\title{
Controlled imbibition in porous medium from a soft wet material (poultice)
}

\author{
N. Ben Abdelouahab ${ }^{1,2}$, A. Gossard ${ }^{2}$, C. Marliere ${ }^{3}$, P. Faure ${ }^{1}$, S. Rodts ${ }^{1}$, P. Coussot ${ }^{1}$ \\ ${ }^{1}$ Univ. Paris-Est, Laboratoire Navier (ENPC-IFSTTAR-CNRS), 77420, Champs sur Marne, France \\ ${ }^{2}$ CEA, DEN, Univ Montpellier, DE2D, SEAD, Laboratoire des Procédés Supercritiques et de \\ Décontamination, Marcoule, 30207 Bagnols-sur-Cèze, France \\ ${ }^{3}$ IFP Energies nouvelles, 1 et 4 avenue de Bois-Préau, 92852 Rueil-Malmaison, France
}

\begin{abstract}
We provide a first approach of the mechanisms of liquid imbibition in a porous medium from a wet paste in contact with this substrate. Through Magnetic Resonance Imaging (MRI) we first show that, in contrast with intuition, the liquid can invade the substrate even if it has a larger pore size than the paste, which induces a lower capillary pressure in the substrate. This phenomenon happens because the paste can easily shrink. We then show that the imbibition stops when the capillary pressure in the substrate balances the stress needed to further contract the paste. The dynamics of the process then mainly results from the competition of these two effects plus the pressure gradient associated with the liquid flow through the paste. This in particular shows that the liquid penetration in a porous medium, from a poultice in contact with this medium, may be controlled by adjusting the poultice characteristics.
\end{abstract}

\section{Introduction}

Poultices spread over the skin are used in medicine from ancient ages with various possible objectives: cooling, inflammation treatment, absorption (of a substance from the body), penetration (of a substance into the body). Poultices are also used for stain removal from decorative stone surfaces [12]; in that case the poultice is maintained wet during a sufficient time for the staining material to dissolve inside the stone and then diffuse in the poultice. Poultices used for the desalination of historical heritage buildings [3-4], are typically pastes made of a mixture of cellulose and/or clay with water, applied to the wall which is to be cleaned. Some water is then soaked in the porous wall where it can dissolve salts. In the next stage, the poultice dries, essentially by water evaporation from its free surface [5], which tends to extract the saline solution from the wall.

In these different applications a phenomenon playing a major role is the imbibition of liquid, from the poultice, to the porous medium in contact with it. Capillary imbibition of some liquid amount in a porous medium is a phenomenon encountered in a huge number of applications, going from the sponge imbibition to the ink penetrating paper or water soaked in plants, wood, soils or buildings. The basic way to describe the process is the Washburn model, which assumes that a straight front saturating the medium, progressively penetrates the medium thanks to a driving force due to the Laplace pressure along the liquid-air interface at the front. This typically leads to a square root of time dynamics of the penetration length of the front [6-7]. For an upward imbibition process the front advance slows down and finally stops at a critical height associated with the balance between gravity and capillary force [7]. These basic characteristics are used in various fields for estimating the permeability of porous media. The detailed features of imbibition nevertheless often slightly differ from those expected from this theory which relies on an average value of capillary force and thus ignores the details of wetting effects at the pore scale. This led to studies of the rough shape of the front in the context of propagation of fluid-fluid interfaces in disordered media [8]. It was also shown that over long times the Washburn prediction for the dynamics fails, with a slow imbibition process beyond the maximum height expected from gravity-capillarity balance [9-10], an effect associated with a change in the mechanism of advance of the meniscus [10]. Another regime change was observed for imbibition in sponges, which was associated with the existence of two pore sizes [11]. At last, the development of a saturation gradient (instead of an abrupt liquid-air interface) was observed for some 
pore shape distribution [12]. Besides, for imbibition (along layer direction) in multi-layered (of different pore sizes) porous media, original features were observed, in particular due to the interplay between the flow characteristics in adjacent layers [13].

Actually, there is a fundamental difference between this process and the imbibition of liquid from a poultice. In the Washburn model and subsequent assumptions behind most studies, the capillary pressure in the liquid source in contact with the porous medium is around zero, i.e. the liquid-air interface is assumed to be planar, so that the driving force is just the Laplace pressure at the front, possibly damped or fostered by gravity effects. When the liquid source is not a bath of pure liquid but instead a porous medium, e.g. a poultice, the situation is more complex since the imbibition implies the extraction of water from this porous source.

In the field of cultural heritage the intuitive belief is that imbibition will occur essentially when the pore size in the wall is smaller than the size of at least some range of pores of the poultice, which will empty to fill the wall pores. This conclusion is supported by MRI observations of the transfers between two solid porous media in contact [14-15]. However, it was already remarked that the water contained of a cement paste in contact with an initially dry bed packing (with larger pores) can be extracted by capillary effects so as to finally saturate the bead packing [16]. These observations were carried out by NMR (Nuclear Magnetic Resonance) on bead packing samples surrounded by paste. This suggests that there exists some physical phenomenon which allows a soft material to supply liquid in a porous medium of larger pores. More generally, this suggests that the whole dynamics of penetration should be described with tools differing from the standard approach.

The objective of the present work is to observe the dynamics and understand the underlying mechanisms of the imbibition in a porous medium when the liquid is initially contained inside a paste in contact with this medium. In that aim we will mainly use Magnetic Resonance Imaging showing directly how the liquid penetrates in the porous medium. This will make it possible to understand the mechanisms and further study the impact of different parameters through simpler (macroscopic) tests. We start by describing materials and procedures. Then we start by presenting an experiment with a liquid bath in contact with the bead packing, which will serve as a reference. Experiments with pastes in contact with a bead packing are then presented for different thicknesses and concentrations of pastes, and different bead sizes, and the results are discussed.

\section{Materials and procedures}

\section{Substrates}

Bead packings were made by simply hand packing glass beads. Glass beads were purchased from CVP Abrasif \& Broyage (France) and sieved in different ranges of diameters. The grain size distribution each glass bead samples, measured with laser grain sizer, was essentially in the form of a curve in a relatively narrow range of sizes with a peak around a mean value. Note that the peak level in the representation of Figure 1 depends on the sampling which was not always the same here. Finally we can consider as a first approximation that we have similar grain size distributions around different mean values (see Fig.1). These glass beads were used after a specific cleaning procedure which consists to rinse them successively with isopropanol, ethanol and ultra-pure water. Except when mentioned the bead packing used for all tests presented below corresponds to the smallest diameter (i.e. $65 \mu \mathrm{m}$ ). 


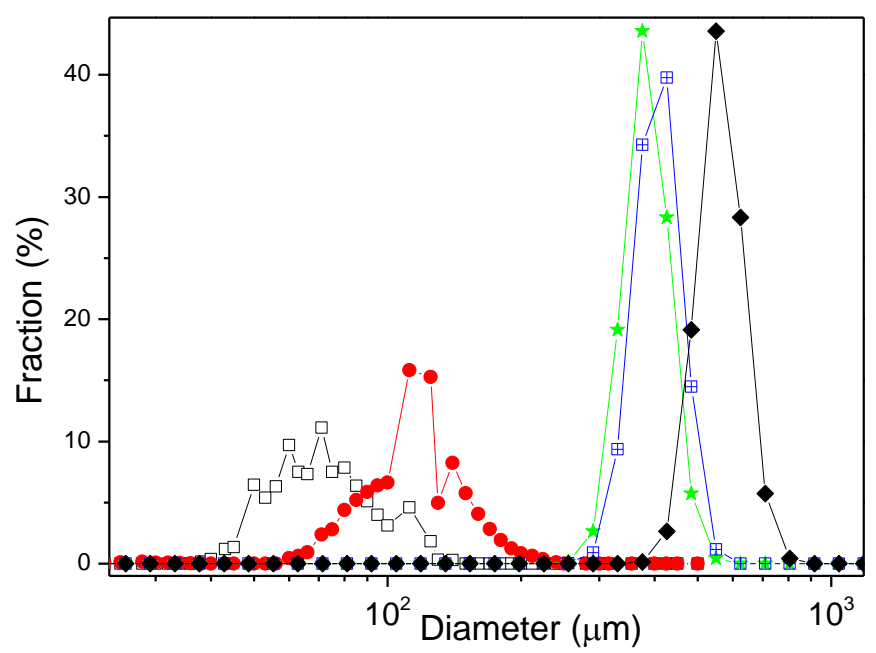

Figure 1: Grain size distributions of the different bead sample used. Mean diameter: (from left to right) $65,125,320,410$, and $560 \mu \mathrm{m}$.

\section{Pastes}

The clay paste is made of kaolinite particles (supplied by Sigma-Aldrich) dispersed in water. Kaolinite is a soft, earthy, usually white, clay mineral, produced by the chemical weathering of aluminium silicate minerals. The kaolinite particles are platelets typically a few $\mu \mathrm{m}$ long. Preparation of kaolin paste was performed by mixing kaolinite (at a weight solid fraction $\varphi$, i.e. the solid to total mass ratio) with distilled water at room temperature for 20 min until obtaining a homogeneous kaolin paste. A mercury intrusion porosimetry test on a dried kaolin paste was carried out in order to get some (rough) view of the pore size distribution of the paste sample. In that aim we let a paste sample dry, so that in its final state it is a rigid porous material with a porosity of $35 \%$. We see (see Figure 2 ) that, in that case, the pore size distribution is mainly in the form of a peak around $0.6 \mu \mathrm{m}$. This gives us the order of magnitude of the pore size in our pastes, which is confirmed by the SEM (Scanning Electron Microscopy) image obtained on a 50\%wt kaolin paste (see inset of Fig.2).

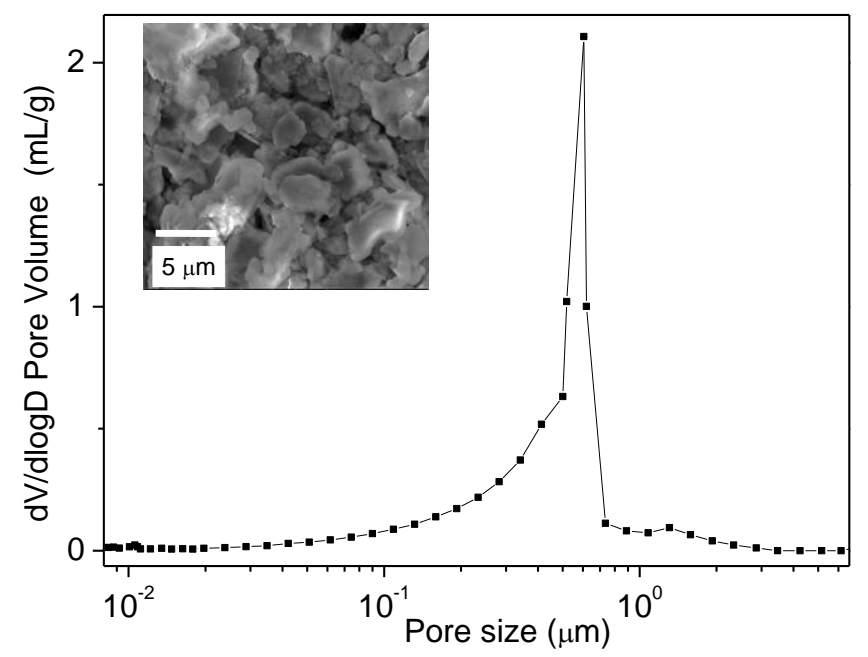

Figure 2: Pore size distribution in a dried kaolin paste investigated by porosimetry: logarithmic variation of the injected specific volume as a function of the pore diameter. The inset shows a SEM (Scanning Electron Microscopy) Image of the kaolin paste (50\%wt) microstructure. 
Rheometrical tests were carried out with TA instrument Discovery HR-1 controlled stress rheometer equipped with rough parallel disks (diameter $40 \mathrm{~mm}$ ) and with a $1 \mathrm{~mm}$ gap. Kaolin pastes appear to be yield stress fluids, which can flow only when the applied stress exceeds a critical value. Their rheological behavior may be appreciated from their flow curves, i.e. the shear stress vs shear rate obtained by imposing a decreasing ramp of shear rates over several decades. The plateau observed at low shear rates corresponds to the yield stress of the material (see typical flow curve obtained for a kaolin paste in the top inset of Figure 3) [17]. An accurate estimation of the yield stress may also be obtained from a creep test, which consists to impose a low shear rate $\left(0.01 \mathrm{~s}^{-1}\right)$ and follow the variation of the stress during the deformation. Typically the stress progressively increases with the deformation, meaning an elastoplastic behavior of the material in its solid regime. Then the stress tends to a plateau at larger deformation, which corresponds to a steady flow in the liquid regime (see bottom inset of Figure 3). In this configuration, the stress level of this plateau is close to the yield stress for a sufficiently low imposed shear rate. The data concerning the yield stress values obtained for different kaolin concentrations are gathered in Figure 3, where we can see the strong increase of the yield stress with $\varphi$.

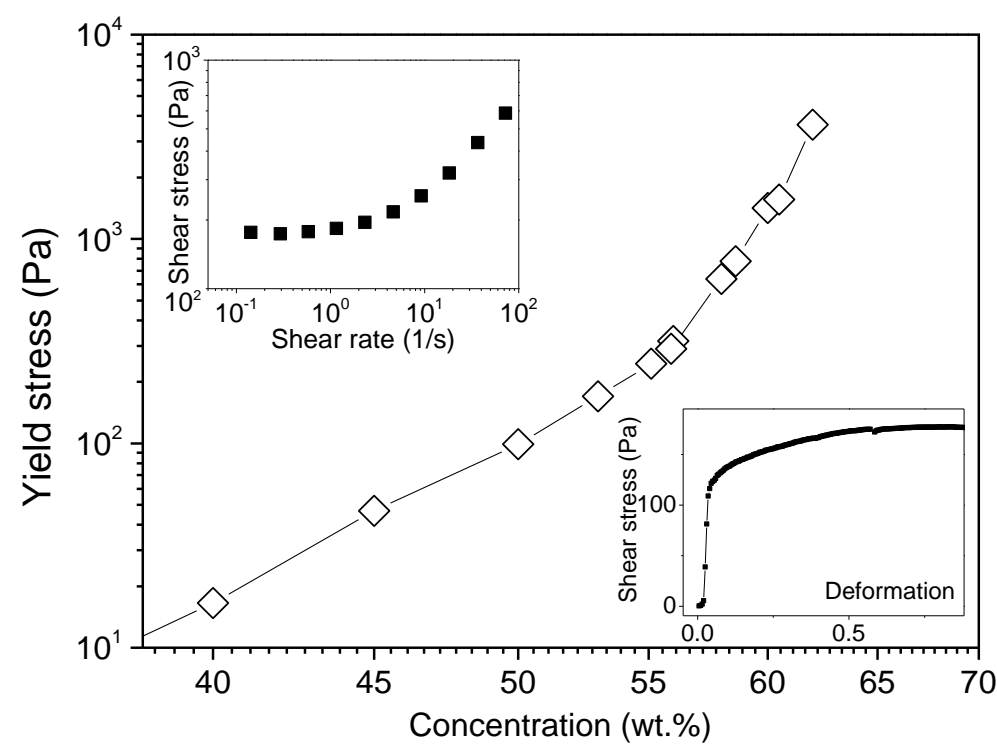

Figure 3: Yield stress of kaolin paste as a function of solid fraction. The top inset shows the flow curve for a concentration of $53 \% w t$ and the bottom inset a creep test under imposed shear rate (see text) for the same concentration.

Sunflower oil from Carrefour (France) was used for some imbibition tests. This oil has a density equal to $0.92 \mathrm{~g} . \mathrm{cm}^{-3}$, a viscosity of $0.06 \mathrm{~Pa} . \mathrm{s}$, and a surface tension around $31 \mathrm{mN} . \mathrm{m}^{-1}$.

\section{Set up}

The paste was coated over the substrate surface (see Fig.4b) so as to form a layer of approximately uniform initial thickness $H$. Note that two small tubes were inserted through the paste and the substrate to evacuate the air pushed by the penetration of the fluid in the bead packing (see Fig.4b). Two tubes were used as security to ensure air evacuation if for some reason one of them was blocked. 
The sedimentation of these kaolin pastes was negligible over the duration of the experiments. We could check that by MRI tests on the paste lying over a solid surface (instead of the porous substrate): no water layer at the top surface of the sample could be detected.

For the tests with oil, the liquid was initially poured over the porous medium (i.e., the sample), then it progressively flowed through it. The sample is simply lying at the bottom of a larger container so that when the liquid arrives at the sample bottom, it starts to fill the container (see Figure 4a). This set-up is put in the center of the magnet of our MRI device, which makes possible the follow of the liquid distribution in the sample with time.

(a)

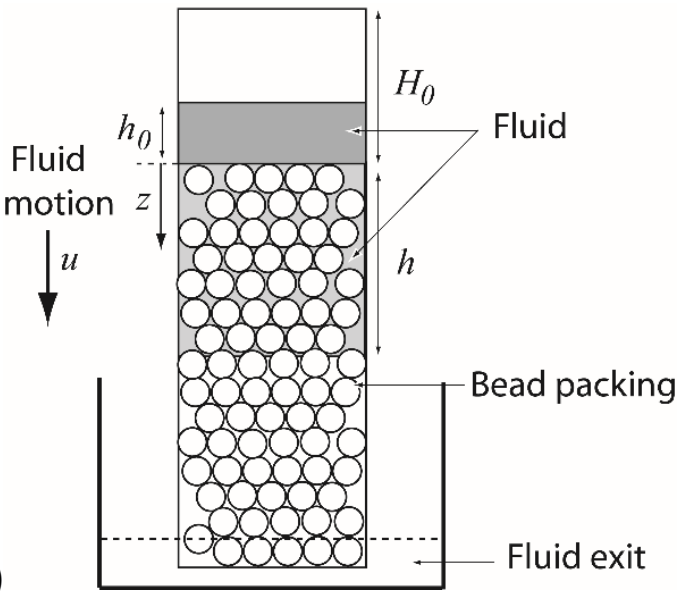

(b)

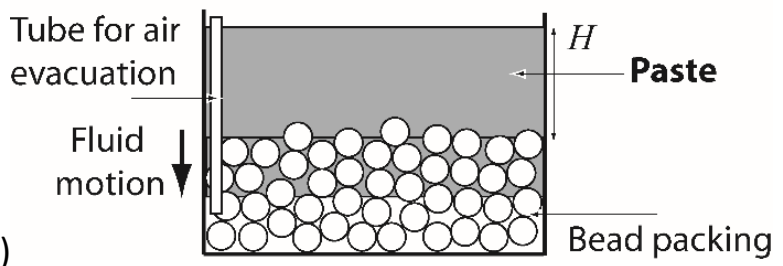

Figure 4: Schemes of the experimental set-up for the soaking experiments: (a) liquid (oil) imbibition, (b) imbibition of water from paste. Note that in this scheme the beads are represented at a much larger scale than their real size.

\section{MRI Measurements}

We looked at the evolution of the water distribution in time with the help of MRI (Magnetic Resonance Imaging) measurements, either from qualitative 2D images of a sample cross section along the vertical axis, or from quantitative 1D projections of the liquid content on the vertical axis, hereunder referred to as 1D profiles, where each data point represents the integrated NMR signal (proportional to the water mass) in a $1 \mathrm{~mm}$ thick layer.

All measurements were performed with a 24/80 DBX 0.5-T ${ }^{1} \mathrm{H}$ MRI spectrometer by Bruker $(20 \mathrm{~cm}$ open diameter in the sample area), operating at $20 \mathrm{MHz}$. During the whole imbibition process, which typically lasts less than one hour, the sample was simply left in the magnetic center of the MRI system.

\section{Profile measurements}

The distribution of apparent water along the sample vertical axis $(z)$ were measured by means of the Single Point Imaging (SPI) sequence [18-19]. Among some other purposes such as removing susceptibility and chemical shift artifacts, this sequence is usually dedicated for studying materials with very short spin-spin $T_{2}$ relaxation times. , i.e. typically under $1 \mathrm{~ms}$. It was chosen here instead of the more classical spin-echo sequences because small relaxation times were likely to occur in our samples at beginning of the imbibition process, when the water amount was small.

Our implementation follows that of [20], which allows the recording of a series of NMR profiles as a function of $\tau$ over some time interval. The signal is further extrapolated based on an exponential 
model to provide a profile $s_{0}$ compensated for the effect of spin-spin relaxation and possibly field inhomogeneities: $s(\tau)=s_{0} \exp \left(\tau / T_{2}^{*}\right)$. Although the monoexponential relaxation can only be an approximate hypothesis, this type of extrapolation can however be shown to provide a reliable estimate of the amount of water present even in slight multiexponential cases. We then yield the distribution of water in small cross-section layers ( $1 \mathrm{~mm}$ thickness) along the sample axis at different positions and time.

The sequence tuning used a field of view of $6 \mathrm{~cm}$ and 60 pixels that is a space resolution of $1 \mathrm{~mm}$. The duration of the hard Radio Frequency pulse was $10 \mu \mathrm{s}$, yielding a tilt angle for the spins of $\theta=8^{\circ} .30$ values of $\tau$ were recorded between $\tau_{\min }=300 \mu \mathrm{s}$ and $\tau_{\max }=590 \mu \mathrm{s}$. The recycling time TR was set to $500 \mathrm{~ms}$, to encompass the long relaxation times observed at the end of imbibition. The whole profiling process was repeated 16 times on purpose of phase cycling and signal accumulation, and lasted about $8 \mathrm{~min}$.

Note that the measured profiles do not simply indicate the water amount, but are also sensitive to the geometry of the sample and its container. In particular, since the bottom of the container and the free surface of the sample are not perfect horizontal planes, the profiles, which record the amount of liquid in thin successive horizontal layers, exhibit some ramp at their edges, , i.e., a progressive decrease of the NMR signal over the millimeter order even if the saturation in the sample is homogeneous.

\section{Imaging}

2D Images were obtained in a vertical cross section along the axis of the sample by means of a spinecho sequence with a slice selection [21]. The measurements are qualitative, and weighted by spinspin relaxation so as to well observe the various parts of the sample. The signal intensity on the MRI picture is therefore not directly proportional to the local water amount, but reads instead $s=s_{0} \times \exp \left(-\mathrm{TE} / T_{2}\right)$. Since $T_{2}$ in a saturating porous medium is a increasing function of the water amount, the image intensity however remains a growing function of the water amount for interpretation.

The sequence tuning used a vertical and horizontal (read out) field of views of $6.5 \mathrm{~cm}$ and $20 \mathrm{~cm}$ respectively, with a space resolution of $1 \mathrm{~mm}$ in both directions. The slice thickness was $1 \mathrm{~cm}$. The whole imaging process was repeated 8 times on purpose of phase cycling and signal accumulation, with $T R=1 \mathrm{~s}$ and $\mathrm{TE}=4.2 \mathrm{~ms}$, and lasted about 9 minutes.

\section{Washburn theory for the vertical imbibition of a simple liquid in a porous medium}

Let us assume that a Newtonian liquid (of viscosity $\eta$ ) flows in direction $\mathrm{z}$ through the porous medium in the form of a homogeneous transversal front progressing at the velocity $u$ (see Figure 2 ). The porous medium is thus saturated (voids filled with water) up to the depth $h$ and dry elsewhere. The height of fluid above the medium is noted. We can apply the Darcy's law to this flow [22-23], i.e. $u=\mathrm{d} h / \mathrm{d} t=(K / \eta) \nabla(p+\rho g z)$ where $p$ is the pressure, $\rho$ the fluid density, $g$ the gravity and $K$ the permeability of the porous medium. At the front, the pressure is equal to the ambient pressure minus the Laplace pressure, which may be written $\sigma \cos \theta / \alpha R$, in which $R$ is the particle radius, $\sigma$ the surface tension and $\theta$ the contact angle. This is finally the capillary pressure, which drives the imbibition process. This expression assumes an average curvature of the meniscus along the liquid-air interface at the front proportional to the bead curvature $(1 / R)$ by a factor $\alpha$ which expresses the size ratio between the meniscus and the bead (which might be about $1 / 5$ ).

Finally we deduce: 


$$
\frac{d h}{d t}=\frac{K}{\eta} \frac{\rho g\left(h+h_{0}\right)+\sigma \cos \theta / \alpha R}{h}
$$

From the conservation of mass we have: $H_{0} S=h_{0} S+h \varepsilon S$, in which $S$ is the section area of the sample, $\varepsilon$ the porosity of the sample, $h_{0}$ the current height of liquid above the bead packing surface and $H_{0}$ the initial height of liquid. Equation (1) may be rewritten as $\mathrm{d} h / B \mathrm{~d} t=(A+h) / h$ whose solution is:

$h-A \ln (1+h / A)=B t$

With $A=\left(H_{0}+\sigma \cos \theta / \alpha \rho g R\right) /(1-\varepsilon)$ and $B=K \rho g(1-\varepsilon) / \eta$. The Lucas-Washburn imbibition [6-7] equation is found for negligible gravity effects $\left(\alpha R \rho g\left(h+h_{0}\right) / \sigma \cos \theta<<1\right)$ :

$h^{2} \approx 2 A B t \approx 2 \sigma K t \cos \theta / \alpha R \eta$

Note that this equation shows that for the same porous medium we expect similar imbibition curves in the form of $h(\sigma t / \eta)$ for different liquids (assuming constant contact angle).

\section{Results and discussion}

\subsection{Imbibition from a pure liquid bath}

We first consider the imbibition of a 65 microns diameter bead packing with oil. Note that for the same test with water, much less viscous than this oil, the speed of the transport was too important and did not allow MRI measurements before the liquid reached the sample bottom.

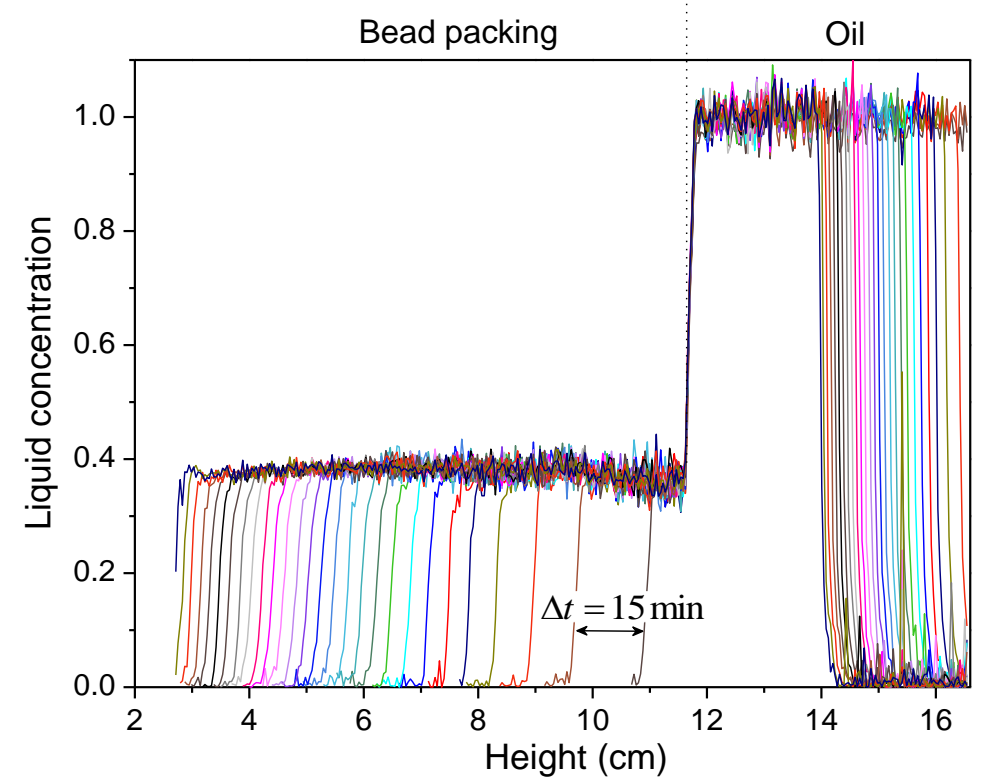

Figure 5: Imbibition of oil in bead packing: successive NMR profiles in time (from right to left). The profiles are represented in terms of "liquid concentration" defined here as the liquid to total volume ratio. The region on the right with saturation equal to 1 corresponds to the pure liquid region, the region on the left corresponds to the bead packing, which is progressively invaded with liquid. The first profile is measured after about $2 \mathrm{~min}$ of flow, the time between the next successive profiles is $15 \mathrm{~min}$. 
Our observations of the process rely on MRI measurements of the successive liquid distributions in time (see Fig.5). In that case we rescaled the NMR signal all along this distribution by the average initial value in the liquid bath, which then provides the saturation distribution along the liquid bath / bead packing system. A concentration of 1 is obviously observed in the liquid bath but we see that the free surface level decreases with time as water penetrates the bead packing. Remarkably, this penetration takes the form of a straight front advancing and saturating the medium, perfectly corresponding to the assumptions of the Washburn model (see Section 2). Moreover, we can observe that the speed of the front advance decreases progressively in time, as shown by the decreasing distance between successive profiles.

From these data we can deduce the amount of liquid which entered the bead packing at any time. This is done by integrating the NMR signal in each region of the system (bead packing and liquid bath). Dividing the liquid volume by the sample cross-section area and the porosity we then get the front depth advance as a function of time (see Fig. 6). This advance follows a square root of time variation as a function of time, as expected from the Washburn model.

We can then compare more completely the predictions of this model with the experimental data. For porous media made of bead packing (at the same packing fraction) with bead diameter $D$ scaling arguments readily lead to the conclusion that the permeability should be proportional to $D^{2}$ : $K=\beta D^{2}$. It was shown [23] that for such materials the scaling constant $\beta$ is around $7 \times 10^{4}$. On the other hand, since the coefficient $\alpha$ in equation (3) is related to the size of the typical meniscus with regards to the particle radius, we can expect it to be of the order of $1 / 5$, but the uncertainty on this value is rather large. Under these conditions, for the imbibition of oil in this bead packing, equation (3) reduces to $h \approx 0.017 \sqrt{t / \mu}$, with $h$ expressed in centimeters, which appears in excellent agreement with the experiment with oil (see Figure 6).

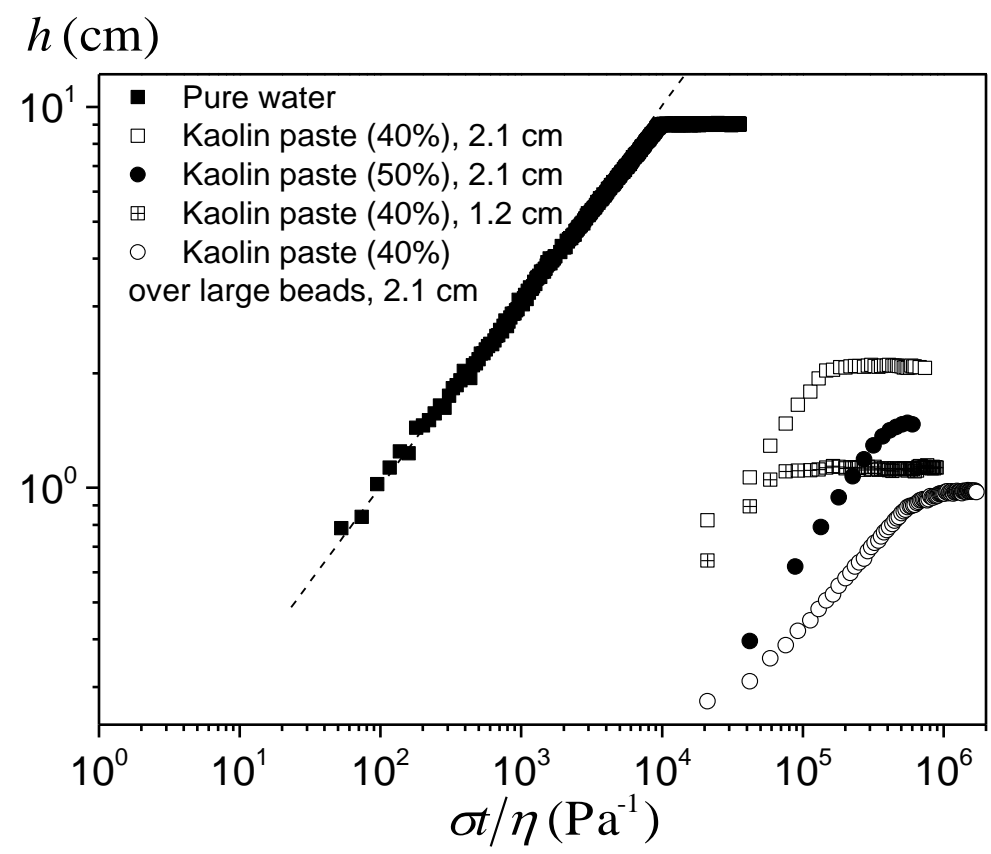

Figure 6: Apparent front depth position as a function of time to viscosity ratio for different materials in contact with a bead packing: oil, kaolin pastes with different initial concentrations and initial thicknesses. The "large beads" mentioned in the legend correspond to the mean diameter of 320 microns. The dotted line is the Washburn model with parameters estimated from the porous structure (see text). 


\subsection{Imbibition from a (wet) paste}

We now place a wet paste over a bead packing (substrate) (see Fig.4b). We start by looking at successive $2 \mathrm{D}$ images of the system to appreciate the process in a global way (see Fig.7). First we can observe that the water penetrates the substrate and we clearly distinguish a front of advance of the liquid (see Fig.7a,b). However, in contrast with the Washburn model assumption, the substrate is not fully saturated behind this front: the color of the wet region of the substrate will turn progressively from a dark blue to a lighter blue in the last images, meaning that the water concentration increases. As the front progresses and finally reaches the sample bottom, the paste shrinks, as shown by the fact that the apparent thickness of the paste region decreases (see Fig.7a,b,c,d). Then the saturation keep increasing in the substrate, while the paste still shrinks. Note that the imbibition is homogeneous, i.e. no significant saturation gradient appears in the substrate in the radial direction. On the other side, the water fraction in the paste seems to decrease faster at the periphery of the sample than in the center, which suggests some edge effects, but this difference remains limited and finally tends to disappear (see Fig.7d). As a whole we have essentially a 1D transfer from the paste to the substrate, so that the 1D NMR profiles are relevant to describe the process in detail.

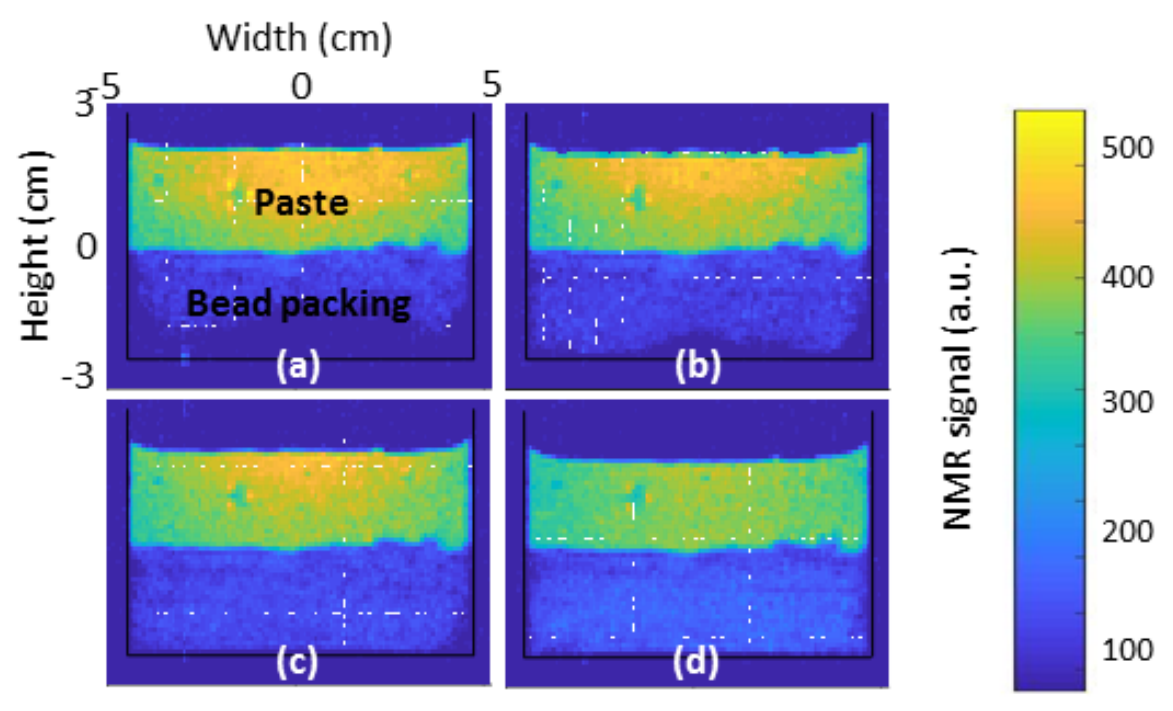

Figure 7: Typical aspect through 2D images of the water imbibition water from a paste to a bead packing in contact at different times: (a) $10 \mathrm{~min}$, (b) $21 \mathrm{~min}$, (c) $54 \mathrm{~min}$, (d) $121 \mathrm{~min}$. The paste is a kaolin paste (initially at $50 \% \mathrm{wt}$ ). The color scale shown on the left corresponds to the NMR signal intensity, i.e. the concentration of water.

The general characteristics of imbibition from paste to bead packing qualitatively observed from the $2 \mathrm{D}$ images also appear in the 1D profiles in time (see Figs. 8-9). The water front penetrates through the all substrate and then, the saturation increases progressively while the paste keep shrinking. The paste shrinkage is associated with both the decrease of the level of its free surface and the decrease of the NMR signal in the substrate region (see Figs. 8-9). Indeed, water extraction leads to a shrinkage and a higher solid concentration in the remaining paste volume.

Now we can examine qualitatively the impact of different conditions, such as paste / substrate thickness ratio, initial paste concentration and substrate pore size. A more quantitative analysis will be provided below in the paper. Two very different cases are observed: 
i) The substrate is saturated at the end of the test (see Fig.9). This means that the power of extraction of the substrate was sufficiently large with regards to the resistance of the paste to water extraction and the available water amount in the paste was sufficiently large to fill the substrate. This situation is reached for either sufficiently small pores, sufficiently large paste / substrate thickness ratio, or sufficiently small solid fraction in the paste. This case just provides an upper bound of some equilibrium conditions (see below).

ii) At the end of the test, i.e. when no more liquid motion is observed, the substrate is not saturated (see Fig.8). However, in any case, there is still some liquid available in the paste for possibly increasing the saturation in the substrate. This final state, with liquid in both regions, means that some equilibrium has been finally reached. The power of liquid extraction of the substrate is balanced by a sufficient resistance of the paste.

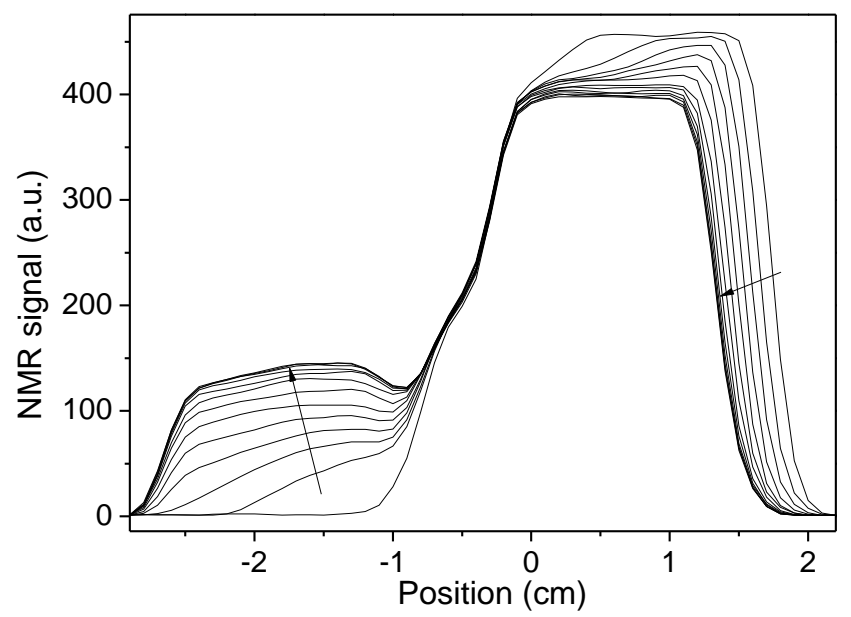

Figure 8: Imbibition of a bead packing from water initially contained in a paste. Initial concentration of the kaolin paste: $50 \%$ wt. Successive NMR profiles in time (from right to left), taken every $11 \mathrm{~min}$.

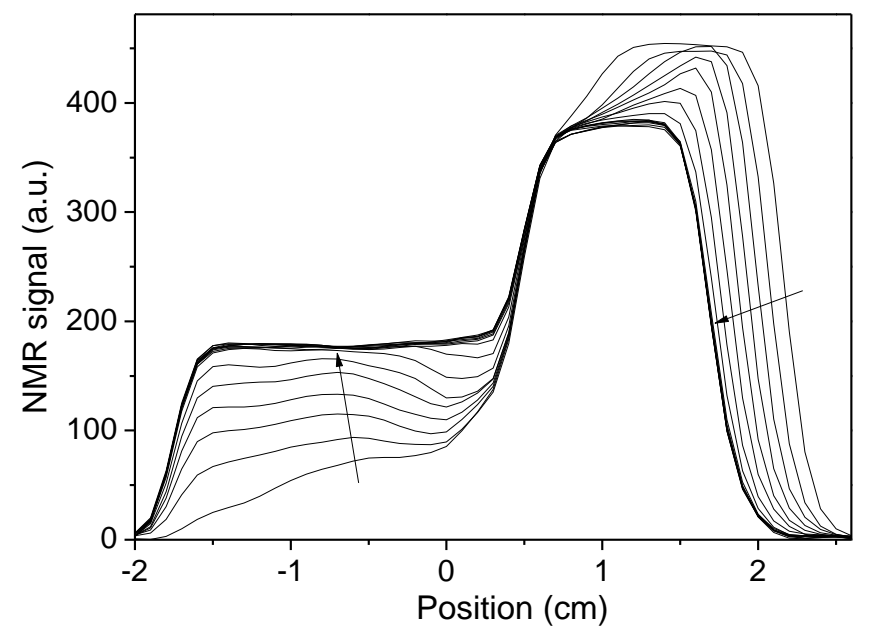

Figure 9: Imbibition of a bead packing from water initially contained in a paste. Initial concentration of the kaolin paste: $40 \% \mathrm{wt}$. Successive NMR profiles in time (from right to left), taken every 4 min $10 \mathrm{~s}$.

In addition, we can remark that in any case: 
- The penetration of water in the substrate is much slower than expected from the Washburn theory (see Fig.6): the dynamics of the imbibition from paste is typically slower by a factor about 1000 with regards to the dynamics expected from the Washburn model (which predicts the same $h(\sigma t / \eta)$ curve for different liquids) ; since the conditions for liquid transport through the substrate are identical, this means that the water extraction from the paste controls and slows down the transport process.

- Even if in the transient stages, the water distribution in the paste is not homogeneous, it systematically ends in a homogeneous state (see Figs. 8-9), which suggests that the equilibrium state concerns the whole (homogeneous) paste sample.

- A remarkable effect, in complete contradiction with the Washburn model assumptions, is that the water penetrates in the form of a "non-saturated" front, i.e. the substrate is not saturated at and behind the front. This suggests that there is a driving force (wetting effect) which leads the liquid inside the medium to form a thin film which would tend to cover the solid surface thanks to wetting properties [24], while the liquid supply (from the paste) and/or the capillary forces are not sufficiently large to fill the sample behind this front. However, the above effect is not general: for larger beads we observe the propagation of a front while the substrate is saturated behind (see Fig.10). Since here the global advance of water is slower, this suggests that the velocity of the thin film at the front would be lower here, an effect for which we have no clear explanation. Finally we leave the possible further study and interpretation of this effect to future work, possibly with a better control of wetting characteristics of the solid surfaces. Under these conditions we propose to simply describe the water amount in the substrate through the mean saturation in this domain, which finally consists to neglect the initial phase of front advance and consider that the substrate essentially fills by increasing its (homogeneous) saturation in time.

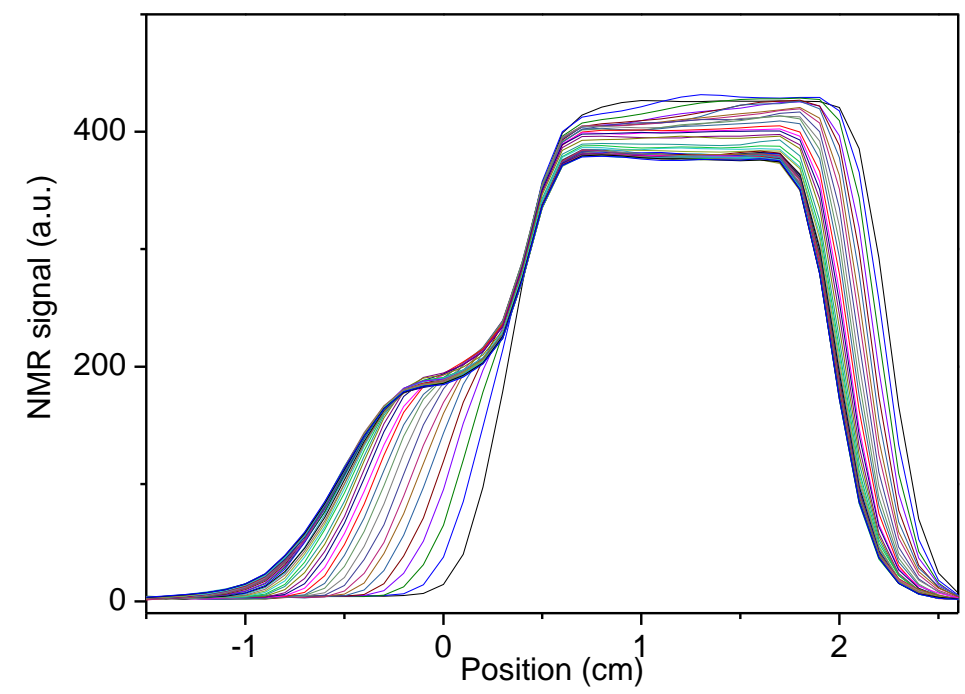

Figure 10: Imbibition of a bead packing (320 microns) from water initially contained in a paste. Initial concentration of the kaolin paste: $50 \%$ wt. Successive NMR profiles in time (from right to left), taken every 8 min.

Finally we can remark that so far we have implicitly considered that only the interstitial liquid phase of the paste penetrated the bead packing. This means that the solid particles of the paste remain as a whole outside of the substrate. On the opposite, we can also consider the possibility of penetration of 
the paste as a more or less homogeneous material (particles + liquid) in the porous medium. Since the paste is a fluid, one could expect that the whole fluid itself would penetrate the bead packing as a result of capillary effects like any liquid in contact with a dry porous medium with sufficiently small pores. However here the situation is more complex because we are dealing with a yield stress fluid, which flows only when the stress applied to it overcomes a critical value. Thus the significant penetration of the paste in the bead packing, which means that it effectively flows through it (i.e. it is not simply deformed in its solid regime), requires that the capillary stress be at least larger than the yield stress of the fluid. According to [23] for the flow of a yield stress fluid through a bead packing the pressure gradient needed to induce a flow through a packing of beads of diameter $D$ is $\nabla p \approx 12 \tau_{c} / D$. Here, if we assume that the capillary pressure has been able to induce a penetration over a distance $x$ (depth of penetration) before stoppage, the pressure gradient for this equilibrium, expresses as $\nabla p \approx 2 \sigma / x R$ (pore radius). We find that, for our basic bead packing (see Section 2), the penetration depth can reach a value larger than $1 \mathrm{~mm}$, and thus be significant within our frame of observation (MRI resolution around $1 \mathrm{~mm}$ ), if the yield stress is smaller than $70 \mathrm{~Pa}$. Considering the yield stress of the paste (see Fig.3) this effect should a priori be significant only for our tests with a paste at a concentration lower than $45 \%$. However, the corresponding flow of the paste through the substrate will be rather slow, so that the extraction of water would likely be faster. This leads to an increase of the solid fraction of the paste, and thus a higher yield stress, making the penetration of the paste as a whole more difficult and so on. This effect likely explains that we do not observe a substantial paste penetration in the bead packing even for this initial concentration. We could check that the concentration increase due to water loss from the paste corresponds to the saturation increase in the bead packing, showing the effect of solid particle paste in the substrate is negligible.

Looking now further at the impact of the different parameters we start by looking at the final state, i.e. the equilibrium reached at the end of the state. In that aim we will look at the final liquid distribution, and we will extract from that the final solid concentration of the paste, by integrating the signal over each region of the composite system to determine the fraction of water in each part. We will also rely on a series of macroscopic tests in which we simply measured the paste concentration at the end of the test. Note that for any of the techniques used here the uncertainty on the concentration is about $1 \%$.

\section{Impact of paste thickness}

We investigate the impact of the paste / substrate thickness ratio, keeping other conditions constant (pore size, paste concentration $(50 \% \mathrm{wt})$ ). In that aim we just maintain the substrate thickness fixed and vary the paste thickness. Obviously more water is extracted from the paste when its thickness is increased and therefore, we get more water in the substrate. However, remarkably, whatever the final saturation in the substrate, the final concentration of the paste is constant: $60.5 \pm 0.2 \%$ for any initial thickness presented on Figure 11. This result is fundamental as it implies that:

- The equilibrium between the extraction power of the substrate and the resistance of the paste corresponds to some intrinsic physical property of the paste, which is related to its solid concentration.

- The extraction power does not vary much with the saturation in the substrate in our range of observation. 


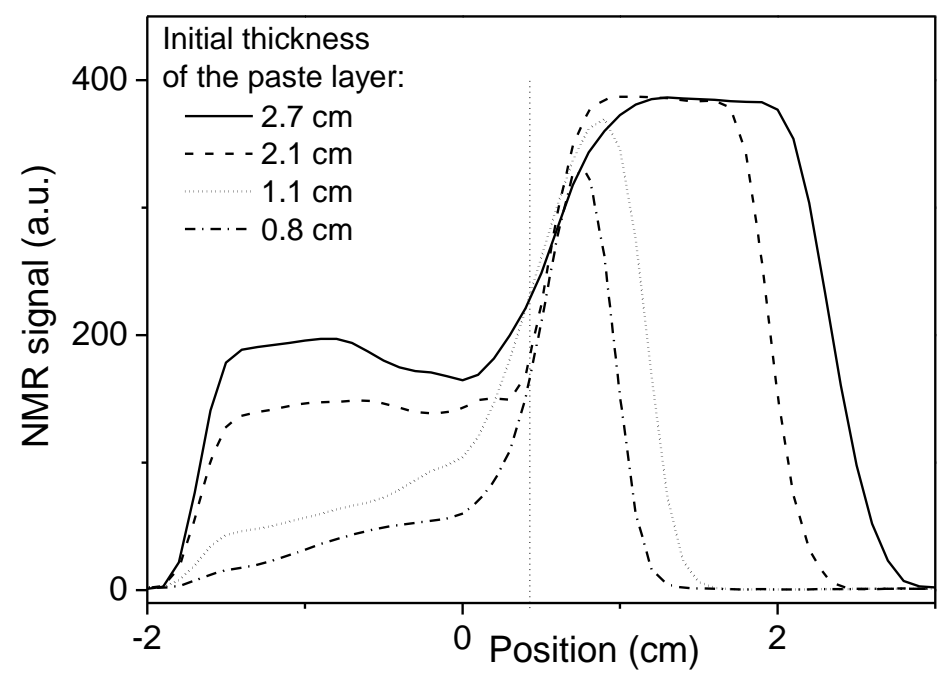

Figure 11: Imbibition of a bead packing from water initially contained in a paste: NMR water profile obtained at equlibrium for different initial thicknesses. Initial concentration of the kaolin paste: $50 \%$.

\section{Impact of the substrate pore size on the paste concentration at equilibrium}

We then test the impact of the pore size, which obviously should affect the extraction power. In that aim we used various bead sizes, ranged from a few tens to a few hundreds of microns, and measured the final concentration of the paste in kaolin after imbibition. It is noteworthy that we maintain all other conditions constant (paste thickness and initial paste concentration). We observe that the yield stress (increasing with concentration) increases with the capillary pressure (see Fig.12) (decreasing with the pore size), which means that the paste concentration at equilibrium decreases when the bead size, or equivalently the pore size, increases.

We know that the yield stress of the paste increases with the concentration due to a denser solid structure. Thus we can assume that the resistance of the paste to shrinkage also increases with its concentration. Indeed shrinking implies some deformation of the solid structure, like a homogeneous flow. For stronger solid porous systems the resistance to shrinkage can be estimated through drained triaxial tests such as those used in soil mechanics [25]. But such tests do not seem to be fitted to soft pastes. Consequently, in order to appreciate this resistance to shrinkage, we suggest to assume here, as a first approximation, that it is proportional to the yield stress. More precisely, since the material is shrunk along the sample axis, the structure undergoes a deformation resembling an elongation. It is generally considered, in particular from the standard 3D constitutive equation of yield stress fluids (see [17]) that the elongational yield stress is proportional to the shear yield stress.

In this way, we determined the yield stress of the paste at the end of each assay, taking care of limiting its deformation before the test. We also measured the yield stress after thoroughly mixing again the sample. The values obtained after mixing are in agreement with those just after preparation (and mixing) of the material, while those for the sample just at the end of the imbibition test are higher (see Fig.12). This means that the process of slow drainage of water from the paste has led to some organization of the structure of the particle network, i.e. the structure is not in a full disordered state as it can be at the end of mixing. 
This phenomenon complicates the establishment of a relationship between the capillary pressure (driving force) and the yield stress of the material (resisting force) at equilibrium. However, it appears that each of these two yield stresses increases linearly with capillary pressure (see Fig.12). This supports our scheme of an equilibrium between the capillary pressure (see Washburn theory Section) and the strength of the particle network of the paste. Actually our results suggest a relationship of the type $\sigma / \alpha R=\xi \tau_{c}$, with here $\tau_{c}$ the yield stress measured on the undeformed paste sample at the end of the test, and $\xi \approx 3.3$ (see Fig.12) a constant factor. This factor appears to be close to that obtained from direct measurement of the elongational yield stress on model yield stress fluids with the help of smooth surfaces [26].

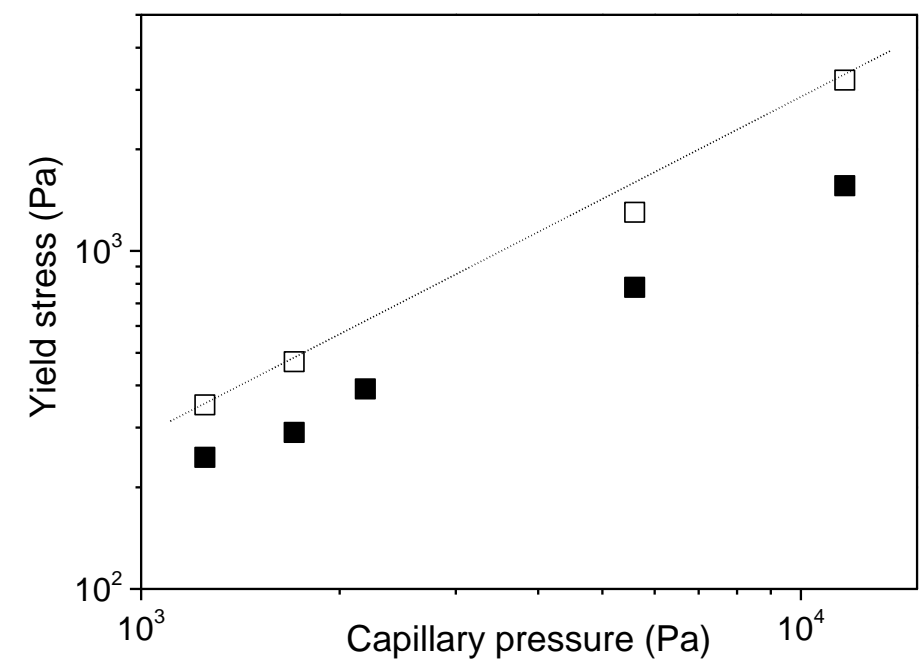

Figure 12: Yield stress of the paste (initially at 50\%) at the end of an imbibition test in contact with bead packing as a function of the capillary pressure of liquid in the bead packing for different pore sizes. The capillary pressure is here estimated as $\sigma / \alpha R$, and thus decreases when the bead radius increases. Yield stress data correspond to measurements carried out with (filled squares) or without (open squares) remixing the sample. The dotted line corresponds to $\sigma / \alpha R=3.3 \tau_{c}$.

\section{Impact of initial paste concentration}

According to the above theory, we would expect no impact of the initial paste concentration in the case of incomplete saturation of the substrate, i.e. when an equilibrium state is reached between the non-saturated substrate and the paste. However this is not what we observe. The final concentration of the paste appears to increase with its initial concentration: for similar other conditions (bead size and paste thickness) we found equilibrium values of $56 \%$ for $40 \%$ initial concentration, $60 \%$ for $50 \%$, and 65 for $60 \%$. It is even more remarkable that it is possible to start a test at the largest concentration obtained at equilibrium for an initial concentration of $50 \%$, and finally get a significant further shrinkage (up to 65\%) when putting this material in contact with a dry bead packing. These observations tend to confirm the effect mentioned in the previous section, that some organization of the kaolin particles leads to some apparent compression yield stress larger than that obtained after mixing, and even larger when this organization has started earlier, i.e. at smaller concentrations.

\section{Dynamics}


The different observations suggest the following mechanisms for imbibition of the bead packing from the paste. As soon as the paste is in contact with the substrate, capillary effects tend to extract the liquid from the paste, and this liquid will then tend to invade the substrate. This process induces a flow of liquid through the paste, and a contraction of the paste, which means that some forces are needed to shrink the paste. Such a situation is typically described within the frame of poromechanics [Coussy]. Here we propose a simpler approach which consists to neglect the possible concentration gradient in the paste, so that we have an equilibrium between three main stresses:

1) The capillary pressure induced by the liquid penetration in the substrate; here we neglect the pressure gradient in the liquid phase in the substrate, which is justified by the fact that the flow is extremely slow so that from the Darcy's law the pressure gradient induced by the flow is small; we also neglect the variation of the capillary pressure with the saturation, simply considering that a constant value is reached as soon as the medium is not saturated. Such a simplified framework is justified by the uncertainty on data and our sole objective here which consists to demonstrate the qualitative consistency of our description. This capillary pressure is the driving force.

2) The resistance to shrinkage of the paste, which is associated with a compression strength. This strength results from the deformation of the solid network of the paste, as is the case in any type of flow of this material. We expect this stress $(K)$ to be proportional to the (shear) yield stress, namely $K(\phi)=\xi \tau_{c}$.

3) The resistance to liquid flow through the shrinking paste. This may be described through the Darcy's law with a permeability $k$ depending on the paste concentration.

We deduce the following pressure balance :

$$
\frac{\sigma}{r}=K(\varphi)+H \frac{\mu}{k(\varphi)} V
$$

In which $V$ is the velocity of penetration of water in the bead packing (flow discharge per unit crosssection), $H$ the current paste thickness, and $\mu$ the liquid viscosity. From (4) we see that we reach the equilibrium when $V=0$, which leads to a relationship between the capillary pressure and the compression strength, in agreement with above observations.

On the other side, during flow we can rewrite this equation as: $\left[\frac{\sigma}{r}-K(\varphi)\right] k(\varphi)=\mu H V$. Since we ignore the variations of the permeability with the paste concentration it is difficult to precisely check the validity of this model. However we can appreciate some aspects of its validity by looking at the variations of $H V$ as a function of the current paste concentration. In that aim we compute the velocity from the mass of liquid successively measured by NMR in the substrate. 


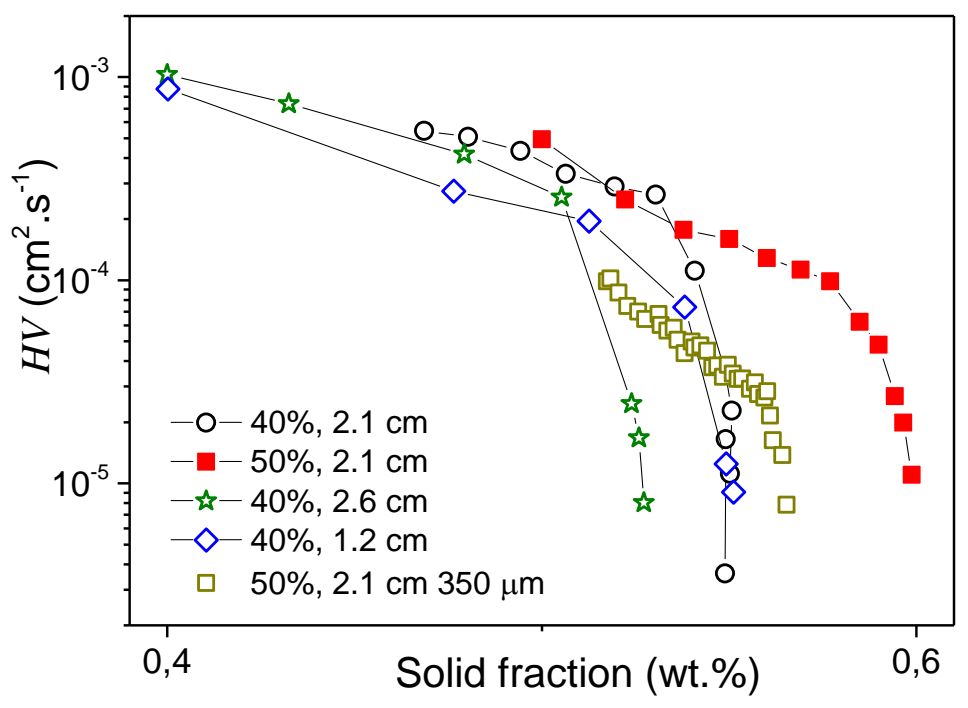

Figure 13: Sample thickness times velocity of imbibition as a function of the solid fraction of the paste for different initial concentrations, paste thickness and bead sizes (see legend).

We can see that these curves are composed of two parts: they slowly decrease over a wide range of concentrations, then they reach a vertical asymptote close to a critical concentration value. This critical concentration corresponds either to the saturation of the substrate or to the partial saturation reached at equilibrium. Not too close to these asymptotes the different data approximately follow a master curve, which confirms the main dependence of $H V$ on the concentration, in agreement with the above model. Moreover the data for the large beads (diameter about six times that of the other data) are situated around a curve situated six times below the above master curve, also in agreement with the equation (4) when the capillary pressure is not too close to the critical compressive stress. We also note the effect of structuration of the paste above mentioned: here it leads to have $H V$ for some initial paste concentration situated above the curves obtained for smaller initial concentrations (see Figure 13).

We can even estimate the permeability of the paste from the data obtained sufficiently far from the asymptote, since in this region the resistance of the structure is negligible compared to the capillary term. For example, at $50 \%$ we find $k \approx 5 \times 10^{-15} \mathrm{~m}^{2}$. Such a value cannot be compared precisely with existing data which in general concern more rigid pasty materials, thus more concentrated kaolin pastes. However we can already see that this value is consistently larger than that obtained at higher solid fraction for the same type of material [27]. We can also remark that such a value is consistent with an estimation through the Kozeny-Carman formula [28] which gives approximately $k \approx R^{2} / 50$ for a bead packing with $R$ the bead radius. Here, using the main pore size $(0.6$ micron) for $R$ we get $k \approx 7 \times 10^{-15} \mathrm{~m}^{2}$, a value of the same order as that found through our tests. Note however that we are here comparing rough estimations in both case, which explains that we can just expect some similar order of magnitude.

\section{Conclusion}


From our results it appears that the liquid transfer from a paste to a porous material is possible even if the pore size of the paste is much smaller than the pore size of the porous medium, under the condition that the paste is sufficiently soft so that it can shrink under a moderate capillary stress. In that case the imbibition process dynamics does not follow a standard Washburn process, it is controlled by the paste characteristics and the pore size of the porous medium. Let us review the detailed characteristics of the process identified in this work:

- Although the pore size of the paste is much smaller than that of the substrate, the liquid is extracted from the paste and penetrates the substrate, while the solid components of the paste remain outside the substrate;

- During all the process, the paste remains saturated;

- The penetration of liquid in the substrate is much slower (by several orders of magnitude) than expected from the Washburn theory which just takes into account capillary effects and substrate permeability; this means that the water extraction from the paste controls and slows down the transport process;

- Under some conditions the liquid penetrates in the substrate in the form of a "non-saturated" front;

- An "equilibrium" is finally reached after some time: it corresponds to either a full or partial saturation of the substrate;

- At equilibrium the liquid distribution in the paste is homogeneous; this means that the equilibrium state concerns the whole paste sample;

- The amount of liquid extracted at equilibrium can be estimated from the balance of the capillary pressure of the liquid in the substrate and the resistance of the paste to shrinkage; this in particular means that there is no direct impact of the pore size of the paste;

- The dynamics of the process can be modelled by taking into account, in addition to capillary pressure in the substrate and resistance to shrinkage of the paste, the resistance to flow of the liquid through the paste.

This means that it is possible to adjust the paste characteristics to obtain the expected imbibition process. This study also opens the way to various new developments since several physical effects observed here still need to be better understood or described, such as the initial imbibition phase in the form of a film, the prediction of the mechanical resistance of the paste during shrinking, the permeability of the paste, etc.

\section{References}

[1] J.A. Lauffenburger, C.A. Grissom, A.E. Charola, Studies in Conservation, 1992, 37, 155-164

[2] S. Spile, T. Suzuki, J. Bendix, K.P. Simonsen, Heritage Science, 2016, 4, 29

[3] A. Bourges, V. Verges-Belmin, Materials and Structures, 2011, 44, 1233-1240

[4] B. Lubelli, R.P.J. van Hees, J. Cultural Heritage, 2010, 11, 10-17

[5] N. Ben Abdelouahab, A. Gossard, S. Rodts, B. Coasne, P. Coussot, Eur. Phys. J. E, 2019, 5, 66

[6] V.R. Lucas, Kolloid Z., 1918, 23, 15-22

[7] E.W. Washburn, Physical Review, 1921, 17, 273-283

[8] M. Alava, M. Dubé, M. Rost, Advances in Physics, 2004, 53, 83-175

[9] T. Delker, D.B. Pengra, P.Z. Wong, Phys. Rev. Lett., 1996, 76, 2902-2905 
[10] M. Lago, M. Araujo, Physica A, 2001, 289, 1-17

[11] J. Ha, J. Kim, Y. Jung, G. Yun, D.N. Kim, H.Y. Kim, Science Advances, 2018, 4, eaao7051

[12] S. Gruener, Z. Sadjadi, H.E. Hermes, A.V. Kityk, K. Knorr, S.U. Egelhaaf, H. Rieger, P. Huber, Proc. Nat. Acad. Sci. , 2012, 109, 10245-10250

[13] S. Ashraf, J. Phirani, Soft Matter, 2019, 15, 2057-2070

[14] H.J.P. Brocken, M.E. Spiekman, L. Pel, K. Kopinga, J.A. Larbi, Materials and Structures, 1998, 31, 49-57

[15] L. Pel, A. Sawdy, V. Voronina, Journal of Cultural Heritage, 2010, 11, 59-67

[16] M. Fourmentin, P. Faure, S. Rodts, U. Peter, D. Lesueur, D. Daviller, P. Coussot, Cement and Concrete Research, 2017, 95, 56-64

[17] P. Coussot, Rheometry of pastes, suspensions and granular materials, Wiley, New York, USA, 2005

[18] S. Emid, J.H.N. Creyghton, Physica B, 1985, 128, 81-83.

[19] M. Bogdan, B.J. Balcom, T.W. Bremner, R.L. Armstrong, Journal of Magnetic Resonance A, 1995, 116, 266-269

[20] P.J. Prado, B.J. Balcom, S.D. Beyea, R.L. Armstrong, T.W. Bremner, Solid State Nuclear Magnetic Resonance, 1997, 10, 1-8.

[21] P.T. Callaghan, Principles of Nuclear Magnetic Resonance Microscopy, Clarendon, Oxford, UK, 1993

[22] S. Whitaker, Transport in Porous Media, 1986, 1, 3-25

[23] T. Chevalier, C. Chevalier, X. Clain, J.C. Dupla, J. Canou, S. Rodts, P. Coussot, J. Non-Newt. Fluid Mech., 2013, 195, 57-66

[24] L.H. Tanner, J. Phys. D, 1979, 12, 1473-1484

[25] J.P. Bardet, Experimental Soil Mechanics, Prentice Hall, New York, USA, 1997

[26] X. Zhang, O. Fadoul, E. Lorenceau, P. Coussot, Physical Review Letters, 2018, 120, 048001

[27] A.S. Michaels, C.S. Lin, Industrial and Engineering Chemistry, 1954, 46, 1239-1246

[28] P.C. Carman, Transactions, Institution of Chemical Engineers, London, 1937, 15, 150-166 